\title{
Chronic obstructive pulmonary disease at the beginning of the XXI Century
}

\author{
Alvar Agusti ${ }^{1,2,3,4}$, Jing Zhang $^{5}$ \\ ${ }^{1}$ Respiratory Institute, Hospital Clinic, Barcelona, Spain; ${ }^{2}$ Institut d'Investigacio Agustí Pi I Sunyer (IDIBAPS), Barcelona, Spain; ${ }^{3}$ Department of \\ Medicine, University of Barcelona, Barcelona, Spain; ${ }^{4}$ Centro de Investigación Biomédica en Red (CIBER) Enfermedades Respiratorias, Instituto \\ de Salud Carlos III, Spain; ${ }^{5}$ Department of Pulmonary and Critical Care Medicine, Zhongshan Hospital, Shanghai Medical College of Fudan \\ University, Shanghai 200032, China \\ Correspondence to: Dr. Alvar Agusti. Respiratory Institute, Hospital Clinic, Villarroel 170, 08036 Barcelona, Spain. Email: aagusti@clinic.cat.
}

Submitted Oct 13, 2019. Accepted for publication Oct 15, 2019.

doi: $10.21037 /$ jtd.2019.10.50

View this article at: http://dx.doi.org/10.21037/jtd.2019.10.50

Chronic obstructive pulmonary disease (COPD) is a major health problem because its high prevalence (about $10 \%$ of the adult population, e.g., $13.7 \%$ in population over 40 year old in China (1), rising incidence, associated morbidity and mortality and personal, familiar, social and economic associated costs (2). Traditionally, COPD has been considered a disease of old men self-inflicted by tobacco smoking. Yet, recent research evidence $(3,4)$ challenges this traditional paradigm. This Editorial briefly reviews some of these challenges and proposes a novel understanding of COPD at the beginning of the XXI Century.

\section{Beyond smoking}

Tobacco smoking is the major environmental risk factor for COPD and every effort must be done to prevent smoking initiation and to favor smoking cessation as soon as possible (5). Yet, solid epidemiologic evidence indicates that about a third of COPD patients worldwide have never smoked (6). Exposure to other environmental risk factors, including biomass fumes, atmospheric pollution and occupational exposure, also increases the risk of $\operatorname{COPD}(3,7)$. Further, as discussed below, there is a number of conditions, including prematurity, poor nutrition and repeated infections during infancy, among others, that may condition the normal development of the lungs, both before and after birth, and lead to COPD in adulthood $(8,9)$.

\section{Lung function trajectories}

After birth, the lungs develop and grow until they reach a peak at about 20 years of age (earlier in females) (5); then, after a brief plateau, lung function declines slightly due to lung ageing (8) (Figure 1). Since the seminal paper by Fletcher and Peto in 1977 (10), we know that, in a proportion of so-called susceptible smokers, lung function decline in adulthood is accelerated, and that this is a major cause of airflow limitation, hence COPD, in late adulthood. Recent epidemiological evidence has confirmed that this actually occurs in about half of patients with COPD diagnosed in the clinic (11); however, the other half have followed a completely different lung function trajectory, characterized by poor lung development in early life (fetal period, infancy and/or adolescence), reduced peak lung function after adolescence and normal rate of lung function decline thereafter (11) (Figure 1). Importantly, these individuals have a higher prevalence and an earlier incidence of cardiac and metabolic co-morbidities, as well as premature death (12). This suggests that an abnormal development of the lung is associated with abnormal development in other organs. This opens new windows of opportunity for prevention, early diagnosis and early treatment (13).

Two other observations are worth discussing here. First, about two thirds of children born with low lung function are able to "catch-up" and regain a normal lung function trajectory, whereas the other third does not (14). Why is it we do not know, but understanding "catch-up" better may help the latter group of children to normalize their lung function and, potentially, to "cure" COPD in adulthood (8). Second, about $10 \%$ of individuals in the general population exhibit a supra-normal lung function 


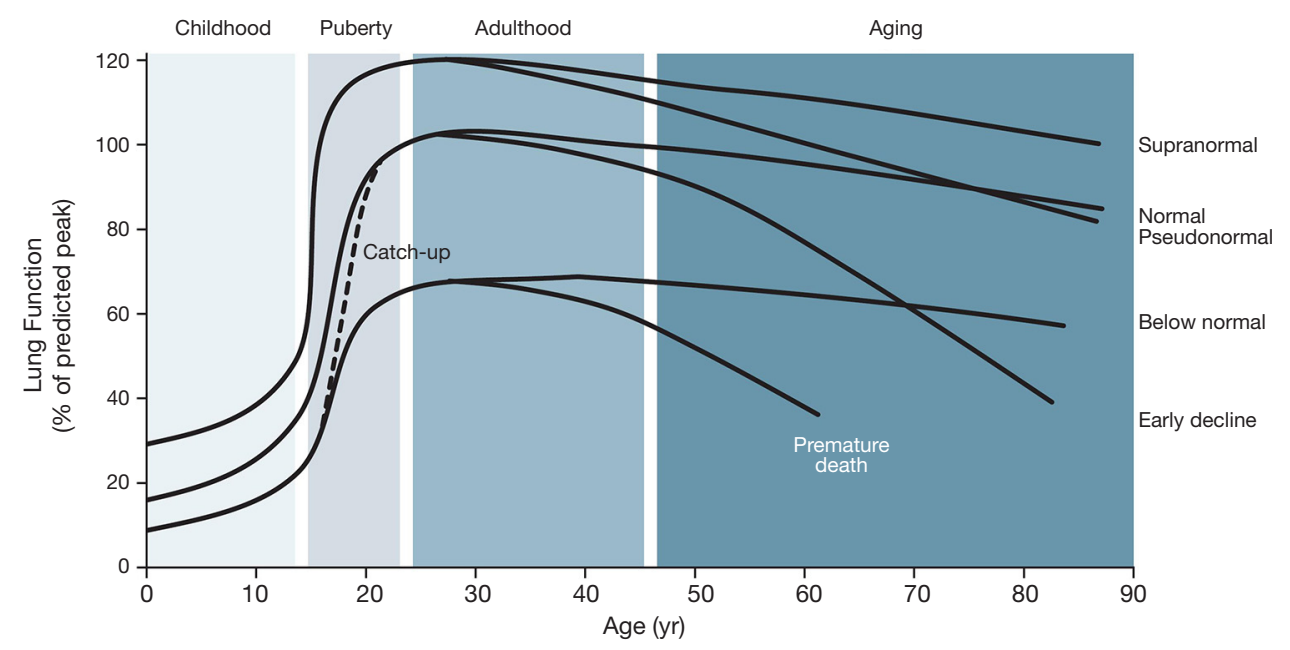

Figure 1 Potential lung function trajectories from birth to death. For further explanations, see text. Reproduced with permission from reference (3).

trajectory (Figure 1) (14). These individuals have a large lung function reserve, so even if the loose a significant part of it during adulthood, they may reach elderly with an apparently normal (pseudo-normal) lung function (3) and contribute to explain why some individuals with emphysema (15) or symptoms (16) may still have normal spirometry.

\section{Disease or syndrome: pre-COPDs}

The current definition of COPD states that it is a "preventable and treatable disease that is characterized by persistent respiratory symptoms and airflow limitation due to airway and/or alveolar abnormalities usually caused by significant exposure to noxious particles or gases" (17). Yet, due to the variety of potential causes and the heterogeneous clinical presentation of COPD (18), COPD should now be understood as a clinical syndrome characterized by varying combinations of chronic respiratory symptoms, structural pulmonary abnormalities (such as airway remodelling, emphysema and/or pulmonary vascular abnormalities), and abnormal lung function (airflow limitation, gas trapping, hyperinflation, reduced diffusing capacity and/or abnormal pulmonary gas exchange), often accompanied by significant extra-pulmonary multimorbidity $(3,4)$. This syndrome results from different, life-long, dynamic, interactive and cumulative gene-environment interactions (smoking and others) that modulate the development, maintenance and function of the lungs (and likely other systemic organs too) (13). This consideration allows a new taxonomy of
COPD (19), where patients with symptoms and structural abnormalities, but without airflow limitation, will be classified as pre-COPD (19).

\section{Addressing the complexity of COPD: endotypes, phenotypes, biomarkers and treatable traits}

COPD is a highly complex and heterogeneous disease. This means that it has several components, that are not always present in all patients (or even in the same patient, through time), and that their relationships are not linear (hence, one cannot be predicted from another one) (20). To address this complexity, the following considerations are relevant (21). First, the term endotype refers to specific biological mechanisms that underlie a given clinical presentation (phenotype), and whose presence can be identified by validated biomarkers $(21,22)$. Second, the term treatable traits refers to those patient characteristics that can be identified by phenotype recognition or biomarker/ endotype determination that merit specific treatment (23). A good example of this approach is the consideration of the number of circulating eosinophils in blood (Eos) to add inhaled steroids (ICS) to bronchodilator treatment in the management of stable COPD, as advocated by the GOLD 2019 (24). Several studies have now convincingly shown that when Eos are higher than 300 cells $/ \mu \mathrm{L}$, ICS are effective in reducing the future risk of exacerbations, whereas they are ineffective when Eos are lower than 100 cells $/ \mu \mathrm{L}$; in between these two thresholds, there may be some effect in some patients (25). Importantly, treatable 


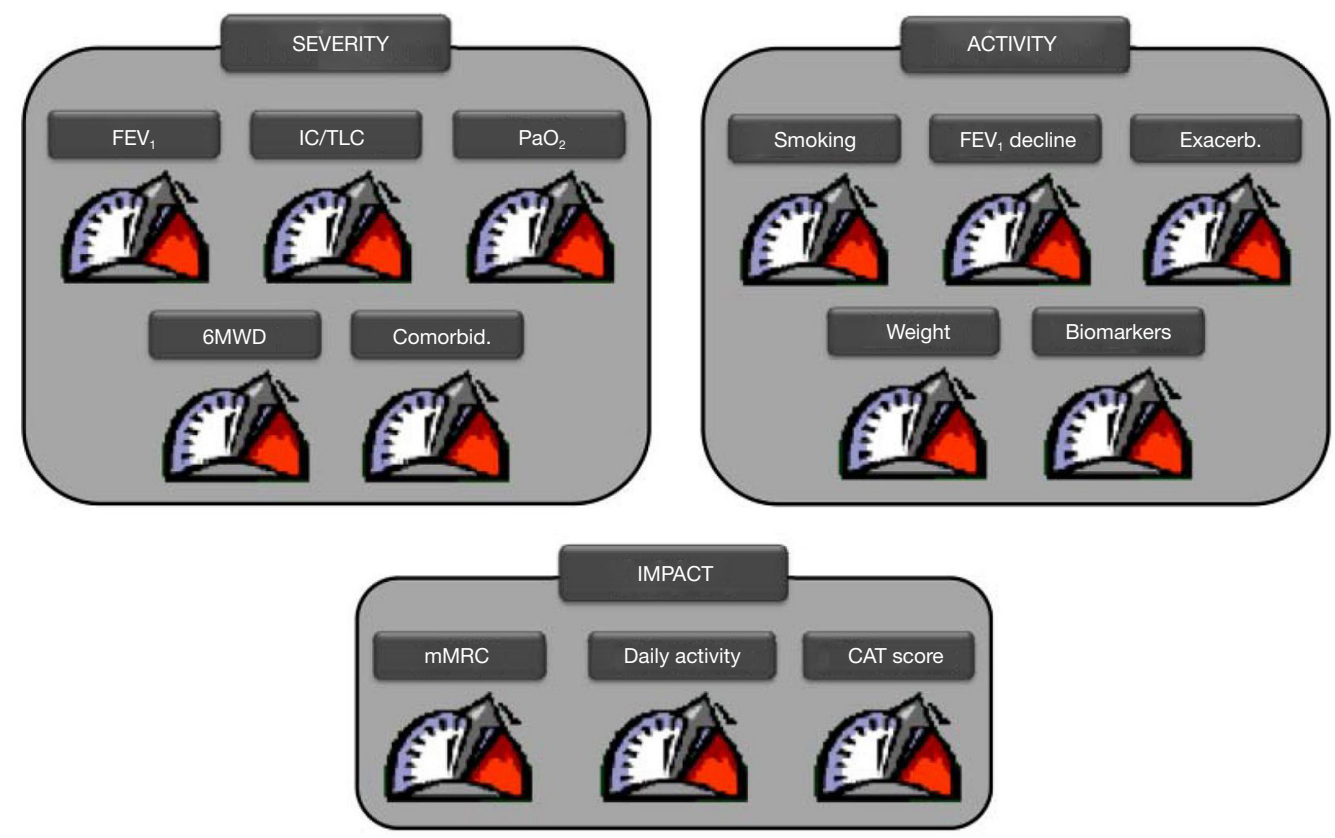

Figure 2 Control panel for the management of patients with COPD. For further explanations, see text. Reproduced with permission from reference (26). COPD, chronic obstructive pulmonary disease.

traits are not restricted to the pulmonary domain, and there are also extra-pulmonary treatable traits (multimorbidity) that require specific treatment if present and, of note, social and behavioural treatable traits (such as smoking, treatment adherence, appropriate use of inhalers and/or living alone, among others) which, albeit not strictly biologically related to COPD, are of extreme importance in its appropriate clinical management $(22,23)$. Finally, it should always be considered that treatable traits may co-exist in the same patient and they may change with time either spontaneously or as a result of therapy (23).

\section{Technology to facilitate clinical practice}

It has been proposed that these treatable traits can be presented to the practicing clinician in the form of a "control panel" (26) (Figure 2), like that used in cars to provide the driver with the important information (speed, engine temperature, fuel reserved, and so on). On the other hand, a free downloadable application of the GOLD 2019 recommendations (24) is now available in the App store (https://apps.apple.com/us/app/gold-2019-pocket-guide/ id1449840740). It intends to facilitate the use of these recommendations in clinical practice.

\section{Conclusions: COPD in the XXI Century}

Many aspects of our understanding of COPD are changing rapidly. They have the potential to modify very significantly the way we prevent, diagnose and treat the disease. The eradication of COPD is ambitious but potentially achievable goal this century (27).

\section{Acknowledgments}

None.

\section{Footnote}

Conflicts of Interest: The authors have no conflicts of interest to declare.

Ethical Statement: The authors are accountable for all aspects of the work in ensuring that questions related to the accuracy or integrity of any part of the work are appropriately investigated and resolved.

\section{References}

1. Wang C, Xu J, Yang L, et al. Prevalence and risk factors of 
chronic obstructive pulmonary disease in China (the China Pulmonary Health [CPH] study): a national cross-sectional study. Lancet 2018;391:1706-17.

2. Halpin DMG, Celli BR, Criner GJ, et al. It is time for the world to take COPD seriously: a statement from the GOLD board of directors. Eur Respir J 2019. doi: 10.1183/13993003.00914-2019.

3. Agustí A, Hogg JC. Update on the pathogenesis of Chronic Obstructive Pulmonary Disease. N Engl J Med 2019;381:1248-56.

4. Celli BR, Wedzicha AJ. Update on clinical aspects of Chronic Obstructive Pulmonary Disease. N Engl J Med 2019;381:1257-66.

5. Kohansal R, Martinez-Camblor P, Agusti A, et al. The Natural History of Chronic Airflow Obstruction Revisited: An Analysis of the Framingham Offspring Cohort. Am J Respir Crit Care Med 2009;180:3-10.

6. Salvi SS, Barnes PJ. Chronic obstructive pulmonary disease in non-smokers. Lancet 2009;374:733-43.

7. De Matteis S, Jarvis D, Darnton A, et al. The occupations at increased risk of COPD: analysis of lifetime jobhistories in the population-based UK Biobank Cohort. Eur Respir J 2019;54:1900186.

8. Agusti A, Faner R. Lung function trajectories in health and disease. Lancet Respir Med 2019;7:358-64.

9. Martinez FD. Early-Life Origins of Chronic Obstructive Pulmonary Disease. N Engl J Med 2016;375:871-8.

10. Fletcher C, Peto R. The natural history of chronic airflow obstruction. Br Med J 1977;1:1645-8.

11. Lange P, Celli B, Agusti A, et al. Lung-Function Trajectories Leading to Chronic Obstructive Pulmonary Disease. N Engl J Med 2015;373:111-22.

12. Agustí A, Noell G, Brugada J, et al. Lung function in early adulthood and health in later life: a transgenerational cohort analysis. Lancet Respir Med 2017;5:935-45.

13. Agustí A, Faner R. COPD beyond smoking: new paradigm, novel opportunities. Lancet Respir Med 2018;6:324-6.

14. Bui DS, Lodge CJ, Burgess JA, et al. Childhood predictors of lung function trajectories and future COPD risk: a prospective cohort study from the first to the sixth decade of life. Lancet Respir Med 2018;6:535-44.

Cite this article as: Agusti A, Zhang J. Chronic obstructive pulmonary disease at the beginning of the XXI Century. J Thorac Dis 2019;11(11):E210-E213. doi: 10.21037/jtd.2019.10.50
15. Alcaide AB, Sanchez-Salcedo P, Bastarrika G, et al. Clinical Features of Smokers With Radiological Emphysema But Without Airway Limitation. Chest 2017;151:358-65.

16. Woodruff PG, Barr RG, Bleecker E, et al. Clinical Significance of Symptoms in Smokers with Preserved Pulmonary Function. N Engl J Med 2016;374:1811-21.

17. Vogelmeier CF, Criner GJ, Martinez FJ, et al. Global Strategy for the Diagnosis, Management, and Prevention of Chronic Obstructive Lung Disease 2017 Report. Respirology 2017;22:575-601.

18. Agusti A, Calverley P, Celli B, et al. Characterisation of COPD heterogeneity in the ECLIPSE cohort. Respir Res 2010;11:122-36.

19. Celli BR, Agustí A. COPD: time to improve its taxonomy? ERJ Open Research 2018. doi: 10.1183/23120541.001322017.

20. Agusti A. The path to personalized medicine in COPD. Thorax 2014;69:857-64.

21. Woodruff PG, Agusti A, Roche N, et al. Current concepts in targeting chronic obstructive pulmonary disease pharmacotherapy: making progress towards personalised management. Lancet 2015;385:1789-98.

22. Agustí A, Bafadhel M, Beasley R, et al. Precision medicine in airway diseases: moving to clinical practice. Eur Respir J 2017. doi: 10.1183/13993003.01655-2017.

23. Agusti A, Bel E, Thomas M, et al. Treatable Traits: Toward Precision Medicine of Airway Diseases. Eur Respir J 2016;47:410-9.

24. Global strategy for the diagnosis, management, and prevention of Chronic Obstructive Pulmonary Disease. Global Initiative for Chronic Obstructive Lung Disease (GOLD), 2019. Available online: www.goldcopd.org

25. Agusti A, Fabbri LM, Singh D, et al. Inhaled corticosteroids in COPD: Friend or foe? Eur Respir J 2018;52:1801219.

26. Agusti A, MacNee W. The COPD control panel: towards personalised medicine in COPD. Thorax 2013;68:687-90.

27. Dransfield M, Stolz D, Kleinert S. Towards eradication of chronic obstructive pulmonary disease: a Lancet Commission. Lancet 2019;393:1786-8. 